\title{
Intraoperative adverse events during laparoscopic colorectal resection-better laparoscopic treatment but unchanged incidence. Lessons learnt from a Swiss multi-institutional analysis of 3,928 patients
}

\author{
P. Kambakamba • D. Dindo • A. Nocito • P. A. Clavien • \\ B. Seifert $•$ M. Schäfer $\cdot$ D. Hahnloser
}

Received: 28 August 2013 / Accepted: 22 December 2013 / Published online: 30 January 2014

(C) Springer-Verlag Berlin Heidelberg 2014

\begin{abstract}
Purpose Intraoperative adverse events significantly influence morbidity and mortality of laparoscopic colorectal resections. Over an 11-year period, the changes of occurrence of such intraoperative adverse events were assessed in this study. Methods Analysis of 3,928 patients undergoing elective laparoscopic colorectal resection based on the prospective database of the Swiss Association of Laparoscopic and Thoracoscopic Surgery was performed.

Results Overall, 377 intraoperative adverse events occurred in 329 patients (overall incidence of $8.4 \%$ ). Of 377 events, 163 (43\%) were surgical complications and 214 (57\%) were nonsurgical adverse events. Surgical complications were iatrogenic injury to solid organs $(n=63$; incidence of $1.6 \%$ ), bleeding $(n=62 ; 1.6 \%)$, lesion by puncture $(n=25 ; 0.6 \%)$,
\end{abstract}

Presentation: The study was presented at the six best paper session of the European Society of Coloproctology meeting in Kopenhagen in November 2011.

P. Kambakamba $\cdot$ A. Nocito $\cdot$ P. A. Clavien

Department of Visceral and Transplant Surgery, University Hospital

Zurich, Zurich, Switzerland

D. Dindo

Department of Visceral Surgery, Triemli Hospital, Zurich,

Switzerland

B. Seifert

Division of Biostatistics, University of Zurich, Zurich, Switzerland

M. Schäfer $\cdot$ D. Hahnloser $(\bowtie)$

Department of Visceral Surgery, University Hospital of Lausanne,

Rue du Bugnon 46, CH-1011 Lausanne, Switzerland

e-mail: Dieter.Hahnloser@chuv.ch

M. Schäfer · D. Hahnloser

Swiss Association of Laparoscopic and Thoracoscopic Surgery,

Zurich, Switzerland and intraoperative anastomotic leakage $(n=13 ; 0.3 \%)$. Of note, $11 \%$ of intraoperative organ/puncture lesions requiring re-intervention were missed intraoperatively. Nonsurgical adverse events were problems with equipment ( $n=127 ; 3.2 \%)$, anesthetic problems $(n=30 ; 0.8 \%)$, and various $(n=57$; $1.5 \%)$. Over time, the rate of intraoperative adverse events decreased, but not significantly. Bleeding complications significantly decreased $(p=0.015)$, and equipment problems increased $(p=0.036)$. However, the rate of adverse events requiring conversion significantly decreased with time $(p<0.001)$. Patients with an intraoperative adverse event had a significantly higher rate of postoperative local and general morbidity (41.2 and $32.9 \%$ vs. 18.0 and $17.2 \%, p<0.001$ and $p<0.001$, respectively).

Conclusions Intraoperative surgical complications and adverse events in laparoscopic colorectal resections did not change significantly over time and are associated with an increased postoperative morbidity.

Keywords Intraoperative adverse event · Intraoperative complication · Colorectal resection · Laparoscopy .

Laparoscopic colorectal surgery

\section{Introduction}

Laparoscopic colorectal surgery has steadily evolved during the last 20 years and is now a valuable approach for the treatment of both benign and malignant colorectal diseases [1-7]. In selected patients with rectal cancer treated by skilled surgeons, laparoscopic surgery resulted in similar safety, resection margins, and completeness of resection to that of open surgery, and recovery was improved after laparoscopic 
surgery. Results for the primary endpoint-locoregional recurrence - are expected by the end of 2013.

At the beginning of this new era of minimal invasive surgery, technical limitations of the equipment and instruments, as well as low levels of personal experience of most surgeons limited the operative indications. Nowadays, it is generally thought that these shortcomings are no longer relevant as the current technology has become very sophisticated, and the surgical community benefits from longstanding experience [8-10]. In addition, there is good evidence that a laparoscopic approach does not impair oncological radicality, and long-term outcome is similar to open surgery [2-6].

Intraoperative adverse events are rarely reported as an entity of their own. Either they are not considered as crucial issues or they are just mentioned as reasons for conversion from a laparoscopic to an open approach [1, 3, 10-12]. Nevertheless, the reported incidence of intraoperative adverse events is ranging up to $20 \%$, and generally, surgical complications, technical problems of the equipment, but also patientrelated anesthesiological problems are summarized as intraoperative adverse events $[12,13]$. While many intraoperative adverse events can be managed without further consequences, some of them are potentially hazardous since they can be the origin of severe postoperative morbidity [14]. These latter ones negatively impact on long-term outcome and represent an important cost factor [15].

The objective of the present trend analysis, based on a large national prospective patient cohort, was to assess the incidence of intraoperative adverse events of laparoscopic colorectal resection during an 11-year period in Switzerland.

\section{Materials and methods}

Data base

The Swiss Association of Laparoscopic and Thoracoscopic Surgery (SALTS) prospectively collect laparoscopic colorectal operations since 1995, whereby 56 (77\%) public hospitals and $17(23 \%)$ private hospitals contribute their patient data [10]. This database is complete, although only about twothirds of Swiss surgeons enrolled their patients undergoing a laparoscopic procedure. More than 120 single items for each patient are documented on a previously designed data sheet and electronically inserted into the data base (Qualicare; Qualidoc, Liebefeld-Bern, Switzerland) [10]. Any missing data were searched by the central data manager responsible for the data base. Conversion was defined as a need for laparotomy, and the decision was up to the individual surgeon.

For the current analysis, all patients undergoing elective laparoscopic colorectal resection from 1995 to 2006 were included. Patients undergoing only diverting colostomies (and no resection) were excluded. The database was closed in 2007.

Definition of intraoperative adverse events

Intraoperative adverse events were defined as any surgical complications directly related to the surgical intervention, as well as any nonsurgical adverse events (not directly related to the surgical act, Table 1) as defined previously by Guller et al. [10]. The responsible surgeon recorded all events from a predefined list immediately at the end of the operation. Outcome measures were defined as previously described [10].

\section{Statistics}

Changes of proportions over time were analyzed using generalized additive models. Dependence on year was adjusted for age, gender, BMI, and ASA score. Model terms of continuous variables are smooth functions with automatic smoothing parameter selection. The analysis was performed using package mgcv in $\mathrm{R}[16,17]$. $p$ values $<0.05$ are considered statistically significant.

Data were analyzed on an intention-to-treat basis, meaning that conversions to an open approach were not excluded from final analysis.

Table 1 Three hundred seventy-seven intraoperative surgical complications and adverse events in 329 patients. Incidence calculated out of 3,928 patients

\begin{tabular}{lll}
\hline & Number & Incidence \\
\hline Surgical complications & 163 & $4.1 \%$ \\
Solid organ lesions & 63 & $1.6 \%$ \\
Puncture lesions & 25 & $0.6 \%$ \\
$\quad$ Stomach/intestine & 7 & \\
Bladder & 7 & \\
Blood vessels & 2 & \\
Solid organs & 5 & \\
Trocar lesion & 4 & \\
Bleeding & 62 & $1.6 \%$ \\
$\quad$ Hematoma/bleeding abdominal wall & 4 & \\
Intraabdominal bleeding & 53 & \\
$\quad$ Hematoma/bleeding requiring transfusion & 5 & \\
Intraoperative anastomotic leaks & 13 & $0.3 \%$ \\
Nonsurgical adverse events & 214 & $5.5 \%$ \\
Problems with equipment & 127 & $3.2 \%$ \\
Anesthetic problems & 30 & $0.8 \%$ \\
Various & 57 & $1.5 \%$ \\
Total & 377 & $9.6 \%$ \\
\hline
\end{tabular}




\section{Results}

Patient demographics and operative data

Between 1995 and 2006, 3,928 patients undergoing elective laparoscopic colorectal resection were enrolled in the study. The median age of patients was 61.5 years (range 15-98), and $58.5 \%$ were women. The median ASA score was 2 (range 1$4)$ and significantly increased over time $(p<0.001)$. The median BMI was $25.8 \mathrm{~kg} / \mathrm{m}^{2}\left(12-50.4 \mathrm{~kg} / \mathrm{m}^{2}\right)$ and increased over time, but not significantly $(p=0.06)$. Of the patients, $18.9 \%$ underwent resection because of colorectal malignancies, and $81.1 \%$ of patients were operated for benign diseases, mainly diverticular disease $(n=2,625 ; 82.4 \%)$. Sigmoid resections were the most common laparoscopic procedure with 2,874 (73.2 \%) cases. Rectal resection, right hemicolectomy, total colectomy, and segmental resections of the transverse colon were performed in 645 (16.4\%), $345(8.8 \%), 40$ (1.0\%), and $24(0.6 \%)$ cases, respectively. Over time, the portion of rectal resections remained stable (range 13.5-23.4 \%). On the other hand, total colectomies were only performed after 2001.

Over the 11-year period, the median postoperative hospital stay was 10 days (range 1-90). The mean rate of conversion was $14.9 \%(n=584)$, and the mean reoperation rate due to an adverse events was $5.0 \%(n=200)$. Interestingly, the majority of surgical revisions $(n=176,88 \%)$ due to complications were managed laparoscopically. Laparotomy was performed in 24 cases. The overall 30-day mortality rate was $0.9 \%$ (35 patients). The majority of colorectal resections were performed by experienced surgeons ( $>100$ total laparoscopic interventions at the time of surgery) in $89.2 \%$ of patients (ranging from in 84.3 to $94.4 \%$ per year). A significant increase of operations performed by less experienced surgeons was recorded during the later observation period from 2001 to 2006 as compared to $1995-2000$ (14 vs. $29 \%$, respectively; $p<0.001)$.

\section{Intraoperative adverse events}

Three hundred seventy-seven intraoperative adverse events occurred in 329 patients (overall incidence of $8.4 \%$ ). Of 377 events, 163 (43\%) were surgical complications, and $214(57 \%)$ were nonsurgical adverse events. Surgical intraoperative complications occurred in $4.1 \%$ of patients and consisted out of solid organ lesions (1.6\%), puncture lesions $(0.6 \%)$, bleeding $(1.6 \%)$, and anastomotic leaks $(0.3 \%)$. On the other hand, the incidence of nonsurgical adverse events was slightly higher (5.5\%) (Table 1). Table 2 lists intraoperative complications and adverse events comparing resections for malignant and benign diseases.

The majority of patients with an intraoperative adverse event $(n=281,85 \%)$ experienced only one complication.
More than two intraoperative adverse events were found in $48(15 \%)$ patients.

The occurrence of a trocar lesion significantly correlated with an additional solid organ lesion $(p<0.001)$ during the procedure. Further intraoperative complications related to equipment problems significantly correlated with the appearance of puncture lesions of the stomach or intestine $(p=0.05)$, puncture lesions of vessels ( $p=0.04)$, trocar lesions $(p<0.001)$, and bleeding of the abdominal wall $(p<0.001)$.

Problems related to the equipment were mainly material dysfunction (i.e., malfunction of stapler, multifunctional devices, misadjustment of camera or monitor). Intraoperative adverse events and complications were comparable between experienced ( $>50$ total colorectal resections) and less experienced surgeons (<total colorectal resections, 8.5 vs. $8.8 \%, p=$ $0.7)$.

Intraoperative surgical complications did not recur frequently

Most of intraoperatively detected and addressed surgical complications did not reoccur postoperatively. Still, most of bleedings and anastomotic leaks (intraoperative anastomotic leak describes a leak at intraoperative testing of the anastomosis, i.e., air insufflation) occurred postoperatively (Table 3).

Six out of $62(9.7 \%)$ intraoperative bleeding complications reoccurred postoperatively. In detail, we observed the reoccurrence of three bleedings in the area of the anastomosis, one case of a migrated clip, and two cases of bleeding from the abdominal wall.

Intraoperatively detected and successfully treated bleeding complications still had significantly increased morbidity as compared to patients without such a complication (36.5 vs. $18.6 \%, p<0.001)$. The same holds true for organ or puncture lesions (39.7 vs. $12.9 \%, p<0.001$ and 48 vs. $18.7 \%$, $p<0.001$, respectively). All 11 intraoperatively missed organ/ puncture lesions required re-intervention (ten surgical revisions and one percutaneous drainage).

Changes of intraoperative adverse events over time

Over time, the rate of intraoperative adverse events did not decrease (Fig. 1; $p=0.231$ ). Surgical intraoperative complications ranged from 1.5 to $6.5 \%$, and nonsurgical adverse events occurred in 5.8 to $17 \%$ per year (Fig. 2). Intraoperative bleeding complications decreased over time (Fig. 3; $p=0.015$ ), whereas problems with equipment significantly increased (Fig. $4 ; p=0.036$ ). The overall conversion rate, but not the conversion rate due to intraoperative adverse events, decreased over time (Fig. $5, p=<0.001$ and $p=0.243$, respectively); $51.2 \%$ (299/584) of conversions were considered reactive conversions as a consequence of an intraoperative adverse event. A reactive conversion was necessary in $40 \%(n=10)$ of puncture lesions, $47.6 \%(n=30)$ of bleedings, $39.7 \%$ 
Table 2 Comparison of intraoperative surgical complications and adverse events in resections for benign and malignant diseases

\begin{tabular}{llll}
\hline & Benign $(n=3,185)$ & Malignant $(n=743)$ & $p$ value \\
\hline Patients with surgical complications & $129(4.1 \%)$ & $34(4.6 \%)$ & 0.52 \\
Solid organ lesions & $50(1.6 \%)$ & $13(1.8 \%)$ & 0.72 \\
Puncture lesions & $19(0.6 \%)$ & $6(0.8 \%)$ & 0.52 \\
Stomach/intestine & 6 & 1 & \\
Bladder & 6 & 1 \\
Blood vessels & 0 & 2 \\
Solid organs & 4 & 1 & \\
Trocar lesion & 5 & 1 & \\
Bleeding & $49(1.5 \%)$ & $13(1.7 \%)$ & 0.67 \\
Hematoma/bleeding abdominal wall & 4 & - & \\
Intraabdominal bleeding & 41 & 12 & \\
Hematoma/bleeding requiring transfusion & 4 & 1 & \\
Anastomotic leaks & $11(0.3 \%)$ & $2(0.3 \%)$ & 0.75 \\
Patients with nonsurgical adverse events & $163(5.1 \%)$ & $51(6.9 \%)$ & 0.45 \\
Problems with equipment & $97(3.0 \%)$ & $30(4.0 \%)$ & 0.17 \\
Anesthetic problems & $20(0.6 \%)$ & $10(1.3 \%)$ & 0.043 \\
Various & $46(1.4 \%)$ & $11(1.5 \%)$ & 0.91 \\
Total patients & $292(9.2 \%)$ & $85(11.4 \%)$ & 0.06 \\
\hline
\end{tabular}

( $n=25)$ of solid organ lesions, $29.1 \%(n=37)$ of technical problems with equipment, and in $3.4 \%(n=9)$ of anesthesiological adverse events. A huge proportion of reactive conversions was performed due to an impaired view (124/ $299(41.5 \%))$.

Changes of postoperative outcome over time

Over time, the overall mean operation time of $180 \mathrm{~min}$ (SD $57 \mathrm{~min}$ ) did not change significantly. Of note, the operation time for converted patients increased significantly over time (from $165 \mathrm{~min}$ in 1995 to $194 \mathrm{~min}$ in 2006, $p=0.01$ ), whereas non-converted patients' operating time decreased (from $199 \mathrm{~min}$ in 1995 to $183 \mathrm{~min}$ in $2006,<0.001$ ).

Hospitalization also significantly decreased by 2 days $(p<0.001)$. Patients with intraoperative bleeding or organ lesions stayed on average 2 days longer in the hospital (10 vs. 8 days).

Overall, postoperative morbidity rate was $18.9 \%$ and mortality was $0.5 \%$. General postoperative complications occurred in $18.9 \%$, and surgical postoperative complications occurred in $13.1 \%$. Both significantly decreased over time $(p<0.001$ and $p=0.033$, respectively). An overview of postoperative surgical complications is given in Table 4.

Intraoperative adverse events influenced postoperative morbidity

Patients with an intraoperative adverse event had a higher postoperative general or surgical morbidity than patients without an intraoperative adverse event (41.2 and 32.9\% vs. 18.0 and $17.2 \%, p<0.001$ and $p<0.001$, respectively).

\section{Discussion}

Intraoperative adverse events occurred in $8.4 \%$ of 3,928 patients undergoing elective laparoscopic colorectal resection and, overall, did not significantly change over time. While bleeding complications decreased, technical problems with the equipment increased.

However, over time, conversion rate and postoperative morbidity decreased, and a higher percentage of surgical interventions were performed by less experienced surgeons.

Table 3 Intraoperative surgical complications and postoperative recurrence

\begin{tabular}{llll}
\hline & Bleeding & Organ/puncture lesion & Anastomotic leakage \\
\hline Detected and treated intraoperatively with recurrence postoperatively & $6(0.2 \%)$ & 0 & $1(0.03 \%)$ \\
Detected and treated intraoperatively with no recurrence postoperatively & $56(1.4 \%)$ & $88(2.2 \%)$ & $12(0.3 \%)$ \\
Not detected intraoperatively with first occurrence postoperatively & $137(3.5 \%)$ & $11(0.3 \%)$ & $131(3.3 \%)$ \\
Total & $199(5.1 \%)$ & $99(2.5 \%)$ & $144(3.7 \%)$ \\
\hline
\end{tabular}


Fig. 1 Percentage of intraoperative adverse events (surgical complications and nonsurgical adverse events) over time $(p=0.57)$

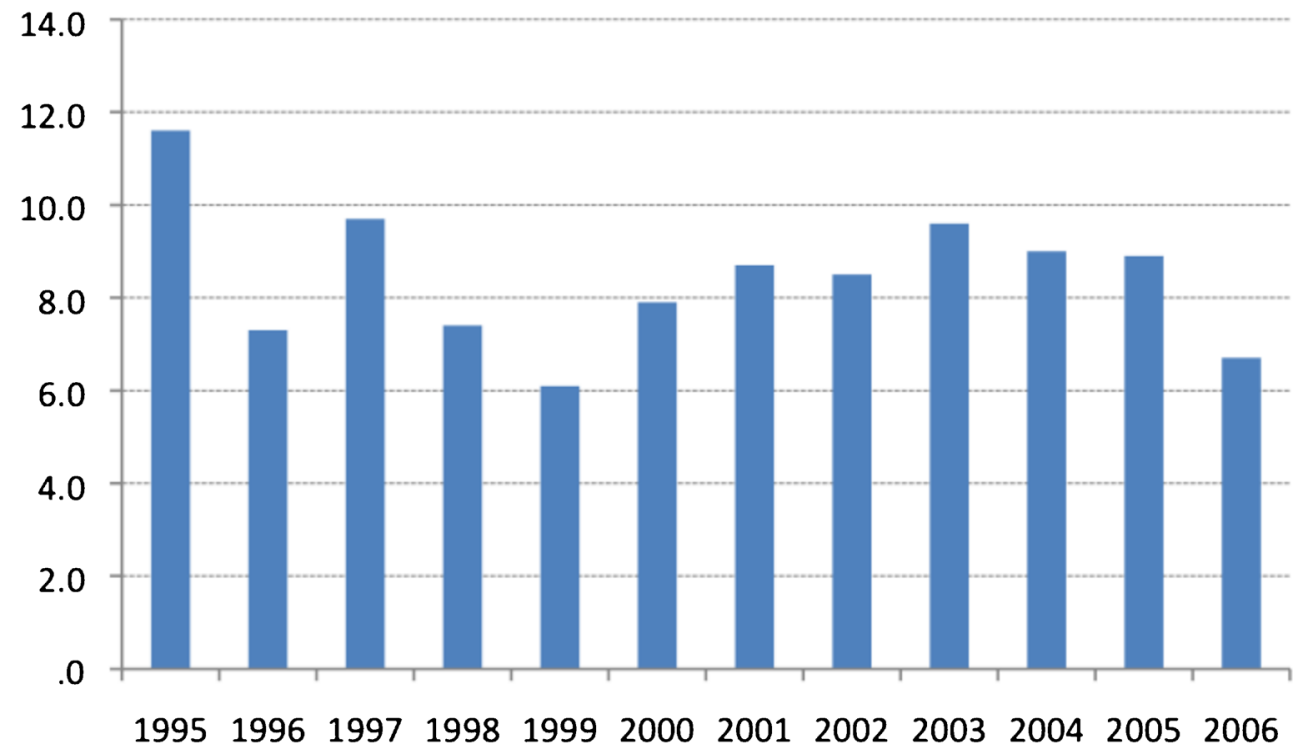

Intraoperative adverse events do not only jeopardize the success of surgical treatment but also strongly influence patients' outcome [14, 18]. Most studies list intraoperative complications and do not distinguish between surgical complications and nonsurgical adverse events. In a meta-analysis including ten RCTs with 2,159 laparoscopic procedures, the mean intraoperative surgical complication rate (defined as hemorrhage, bowel injury, and solid organ injury) was $7.9 \%$ ranging from 0 to $16.1 \%$ [12]. A reason for the lower intraoperative surgical complication rate of $4.1 \%$ in our study might be the smaller portion of oncological colorectal resections (18.9 vs. $96.2 \%$ ) and the special interest in laparoscopic surgery of participating hospitals. However, in another analysis of 1,316 patients, intraoperative surgical complications $(4.5 \%)$ were similar to our study [13]. Only few studies reported on intraoperative complications of open procedures. Intraoperative organ lesion occurred in $1.6 \%$ of open procedures and in $2.4 \%$ of laparoscopic procedures, with bleeding in 2.3 vs. $3 \%$, respectively [10].

Surgical complications are directly related to the surgical act and mainly include bleeding, perforation, and anastomotic problems. In a multicentric trial analyzing 4,834 laparoscopic colorectal procedures, intraoperative bleeding and organ lesions occurred each in $1.7 \%$ [19]. In our study, the rates of bleeding $(1.5 \%)$ and organ lesions $(1.6 \%)$ were comparable to those in the literature. Nevertheless, a portion of intraoperative complications can be missed during surgery. In this study, most of organ lesions were detected intraoperatively, and intraoperative bleeding was successfully managed and reoccurred seldom.
Fig. 2 Percentages of intraoperative surgical complications and nonsurgical adverse events over time

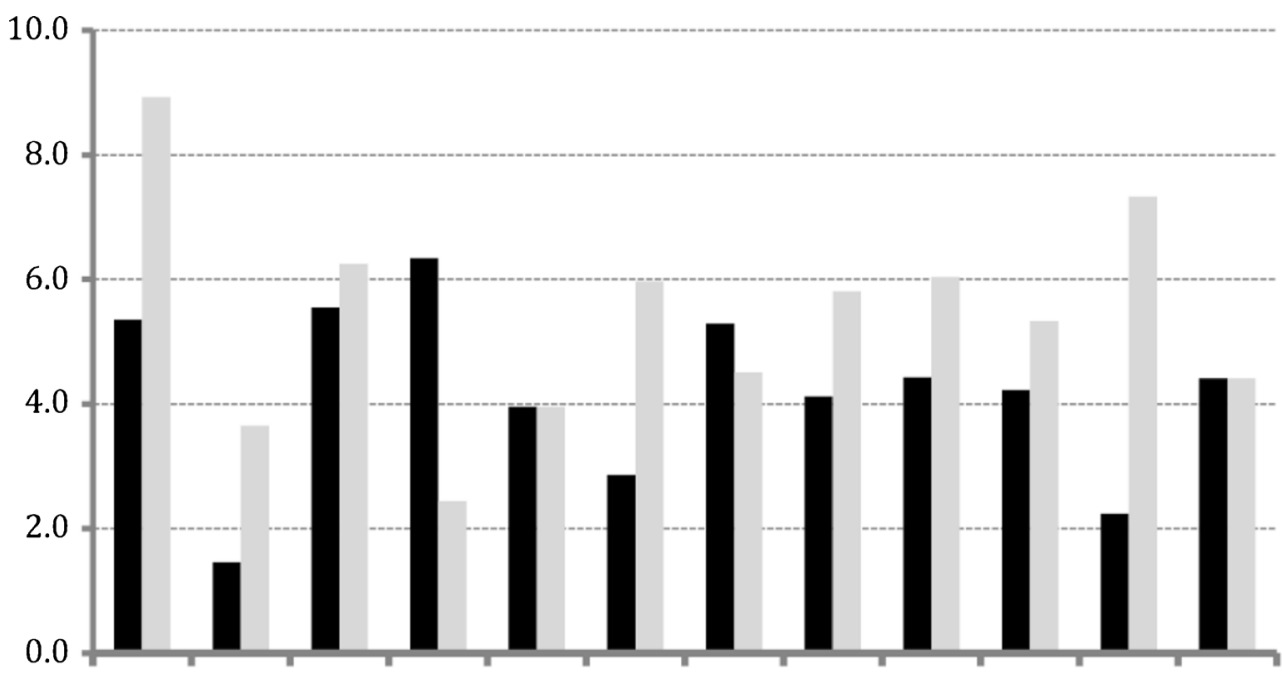

199519961997199819992000200120022003200420052006 
Fig. 3 Percentages of intraoperative bleeding over time $(p=0.015)$

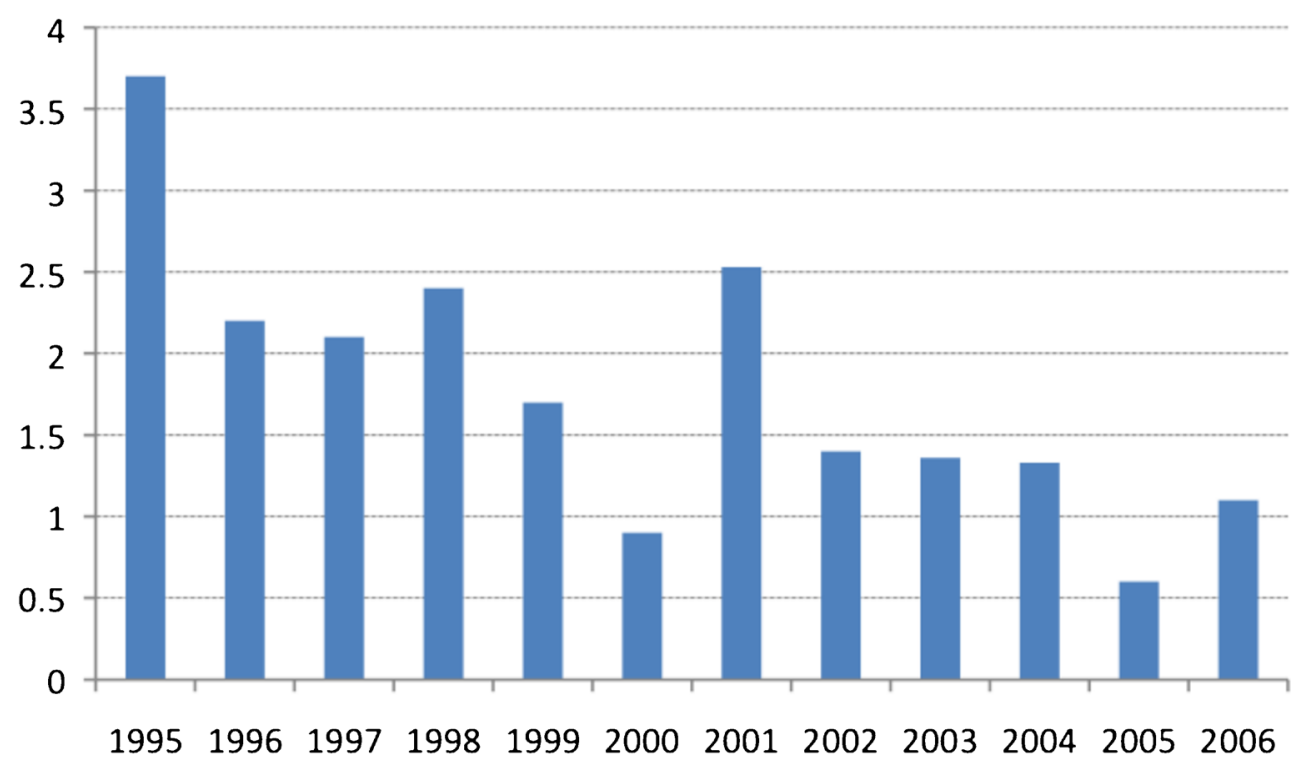

Bleeding complications significantly decreased over time. Several studies suggest that different sealing devices (i.e., Ultracision, Ligasure) are safe and useful instruments for laparoscopic colorectal resections. The harmonic scalpel and the Ligasure were introduced in 1993 and 1998, respectively. Interestingly, the incidence of intraoperative bleedings decreased since 1998. The beneficial effect of these devices on blood loss has been previously described [20-22]. The increasing popularity and utilization of coagulation devices may be one explanation for the significant reduction in bleeding complications.

Surprisingly, equipment problems significantly increased over time and continue to be of concern. Nevertheless, with an incidence of $3.2 \%$ in technical equipment complications, our data is comparable to other studies on that topic [23].

We could not identify a single cause of equipment problem. Stapling issues (e.g., misfiring) were less frequent than problems with the camera or electrical devices. Multifunctional tools such as Ultracision, Ligasure, and others are certainly of benefit to the surgeon, but may also cause problems within the operating room if not correctly handled by the staff. On the other hand, the introduction of reliable vessel sealing devices has certainly contributed to the improvement of patient outcomes [8, 20-22]. The correct handling of these tools remains important, and most companies continue to offer training
Fig. 4 Percentages of intraoperative equipment problems over time $(p=0.036)$

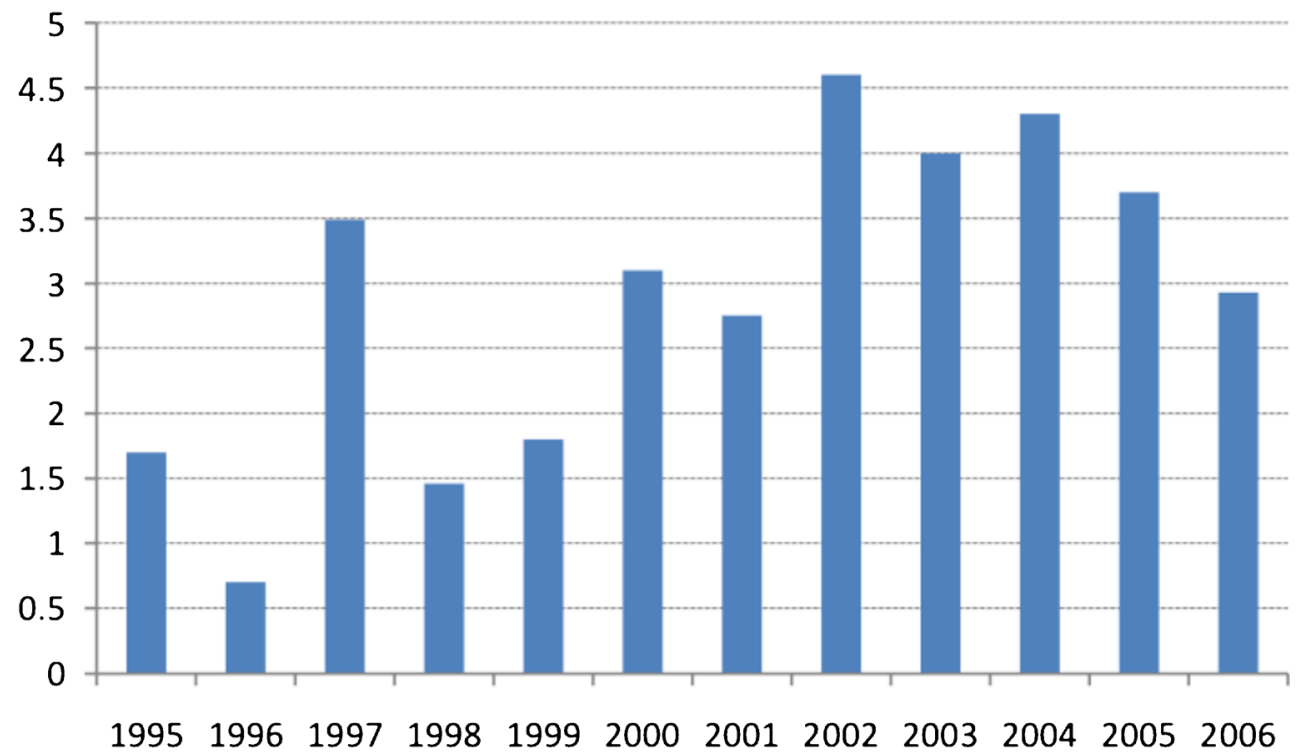


Fig. 5 Percentages of overall conversion rate over time $(p<0.001)$

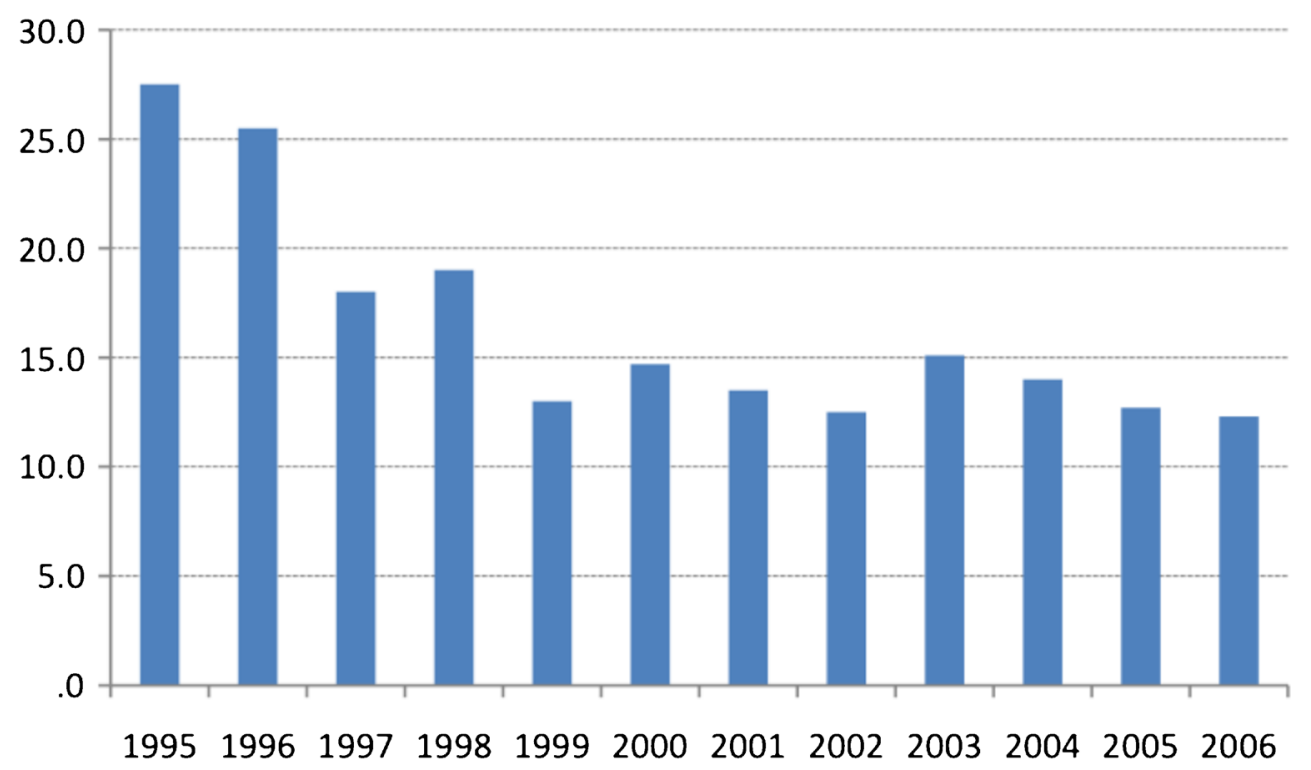

courses for surgeons. However, very rarely dedicated instructions are offered to the non-surgeon personnel in the operating room or during the re-sterilization process, especially in the setting of teaching hospitals with a high turnover and frequent changes during shifts.

Overall, intraoperative adverse events did not change over time even after we corrected for age, gender, BMI, and malignant disease, the most commonly identified factors associated with an intraoperative adverse event [13, 24-26]. However, an intraoperative adverse event significantly increased postoperative morbidity from 17 to $33 \%$ in our study. The tendency to operate patients at higher risk with a higher ASA score may be one explanation for the lack of significant

Table 4 Postoperative surgical complications. Incidence calculated out of 3,928 patients

\begin{tabular}{lll}
\hline Postoperative surgical complications & Number & Incidence (\%) \\
\hline Bleeding & 143 & 3.6 \\
Abdominal wall & 52 & 1.3 \\
Intraabdominal & 49 & 1.2 \\
Requiring transfusion & 42 & 1.1 \\
Infectious surgical complication & 125 & 3.2 \\
Wound infection & 81 & 2.1 \\
Intraabdominal Abscess & 28 & 0.7 \\
Peritonitis & 16 & 0.4 \\
Anastomotic leak & 131 & 3.3 \\
Perforation & 11 & 0.3 \\
Prolonged paralytic ileus & 25 & 0.6 \\
Obstructive ileus & 12 & 0.3 \\
Various & 68 & 1.7 \\
Total & 515 & 13.1 \\
\hline
\end{tabular}

reduction of intraoperative adverse events. Another factor worth being discussed is the increased number of operations performed by less experienced surgeons $(<50$ total colorectal resections) over time. In 2006, the evaluation of an expert survey calculated the surgeon's experience as one of the strongest indicators for intraoperative complications [26]. Experience of $<50$ procedures was associated with a significant increase of intraoperative complications (8.1 vs. $1.7 \%$ ) as described in a multicentric Brazilian trial, analyzing 1,966 patients [27]. However, these findings were not confirmed in a recent study on 991 consecutive laparoscopic resections [28]. Likewise, Langhoff et al. suggested that laparoscopic surgery for colorectal cancer can be performed safely by supervised trainees with good short-term results [29].

The majority of patients in our study were still operated by experienced surgeons ( $>100$ total colorectal resections), $91.3 \%$ (1995-2000) and 88.2\% (2001-2006), respectively. In accordance to the latter studies, our data revealed no significant difference in the rate of intraoperative adverse events between experienced and less experienced surgeons in our data. Therefore, laparoscopic colorectal resections may be safe teaching procedures in an appropriate environment.

Another important finding of our study was the significant decrease in conversion rate.

Similarly, a recent systematic review on laparoscopic rectal resections reported on a strong time trend for conversion rates, which ranged from 0.6 to $32.4 \%$ [30].

Intraoperative bleedings of $47.6 \%$ and organ lesions of $39.7 \%$ were not manageable laparoscopically and resulted in a laparotomy. This is comparable to a study on 926 patients from 2000 to 2007 where intraoperative bleeding (46.7\%) and organ lesions (40\%) were the major reasons for conversion [18]. Although conversion 
is reported to occur in $0-46 \%$, most studies today report on conversion rates for laparoscopic colorectal resections of approximately $12 \%[9,31]$. Conversions remain an important risk factor for postoperative morbidity and mortality of laparoscopic procedures. As impressively demonstrated by Mroczkowski et al., converted rectal cancer patients suffered from significantly more complications than completed laparoscopic cases and planned open surgery patients [32].

A Brazilian multicentric analysis reflected a significant reduction of conversion rate for experienced surgeons (16.6 vs. $6.8 \%$ ) [27]. In our study, operation time decreased over time for all patients $(p<0.001)$; on the other hand, operation time for converted patients significantly increased $(p=0.010)$. This and the fact that intraoperative adverse events were more often controlled laparoscopically, might indirectly indicate that surgeons converted later, and this is without increasing postoperative complications, because surgical and general morbidity also decreased with time.

\section{Conclusion}

The herein study is limited by the fact that the majority (77\%) of participating hospitals had a special interest in laparoscopic surgery. Therefore, a longitudinal observation of the outcome over time in smaller county hospitals would probably give more representative information about the changes over time. Also, the recorded events were not clearly defined, but had to be marked from a given list. Surgeons might have had different interpretations of an event; however, the list of events remained the same over the entire study period. There were several strengths to the study. First, the sample size was large, the statistical power to detect clinically relevant differences was high, and the data were complete (no missing data). Second, the study was population-based and thus had excellent generalizability, despite the limitation by the abovementioned special interest of hospitals in laparoscopic surgery.

Although the study was based on Swiss patients only, the authors believe that the results can be generalized to all countries where the standard of laparoscopic surgery is high.

This study has provided evidence that the incidence of intraoperative adverse events did not change significantly. Hence, surgeons must continue to operate safely as intraoperative adverse events continue to be linked with increased postoperative morbidity. Further, the increasing utilization of technical devices may involve an undeniable risk of increased technical problems. Therefore, regular training and guided instructions for laparoscopic surgeons and the whole involved surgical staff are mandatory.
Conflicts of interest None.

\section{References}

1. Jacobs M, Verdeja JC, Goldstein HS (1991) Minimally invasive colon resection (laparoscopic colectomy). Surg Laparosc Endosc 1: $144-150$

2. Clinical Outcomes of Surgical Therapy Study Group (2004) A comparison of laparoscopically assisted and open colectomy for colon cancer. N Engl J Med 350:2050-2059. doi:10.1056/NEJMoa032651

3. Guillou PJ, Quirke P, Thorpe H, Jayne DG, Smith AM, Heath RM, Brown JM (2005) Short-term endpoints of conventional versus laparoscopic-assisted surgery in patients with colorectal cancer (MRC CLASICC trial): multicentre, randomised controlled trial. Lancet 365:1718-1726

4. Veldkamp R, Kuhry E, Hop WC, Jeekel J, Kazemier G, Bonjer HJ, Haglind E, Påhlman L, Cuesta MA, Msika S, Morino M, Lacy AM, Colon cancer Laparoscopic or Open Resection Study Group (COLOR) (2005) Laparoscopic surgery versus open surgery for colon cancer: short-term outcomes of a randomised trial. Lancet Oncol 6:477-484

5. Yamamoto S, Ito M, Okuda J, Fujii S, Yamaguchi S, Yoshimura K, Sugihara K, Watanabe M, Japan society of Laparoscopic Colorectal Surgery (2013) Laparoscopic surgery for stage 0/I rectal carcinoma: short-term outcomes of a single-arm phase II trial. Ann Surg 258(2): 283-288

6. Asoglu O, Balik E, Kunduz E, Yamaner S, Akyuz A, Gulluoglu M, Kapran Y, Bugra D (2013) Laparoscopic surgery for rectal cancer: outcomes in 513 patients. World J Surg 37(4):883-892

7. Van der Pas MH, Haglind E, Cuesta MA, Fürst A, Lacy AM, Hop WC, Bonjer HJ, Colorectal cancer Laparoscopic or Open Resection II (COLOR II) Study Group (2013) Laparoscopic versus open surgery for rectal cancer (COLOR II): short-term outcomes of a randomised, phase 3 trial. Lancet Oncol 14(3):210-218

8. Hubner M, Demartines N, Muller S, Dindo D, Clavien PA, Hahnloser D (2008) Prospective randomized study of monopolar scissors, bipolar vessel sealer and ultrasonic shears in laparoscopic colorectal surgery. Br J Surg 95:1098-1104

9. Daetwiler S, Guller U, Schob O, Adamina M (2007) Early introduction of laparoscopic sigmoid colectomy during residency. Br J Surg 94:634-641

10. Guller U, Rosella L, Karanicolas PJ, Adamina M, Hahnloser D (2010) Population-based trend analysis of 2813 patients undergoing laparoscopic sigmoid resection. Br J Surg 97:79-85

11. Tekkis PP, Senagore AJ, Delaney CP (2005) Conversion rates in laparoscopic colorectal surgery: a predictive model with, 1253 patients. Surg Endosc 19:47-54

12. Sammour T, Kahokehr A, Srinivasa S, Bissett IP, Hill AG (2011) Laparoscopic colorectal surgery is associated with a higher intraoperative complication rate than open surgery. Ann Surg 253:35-43

13. Kirchhoff P, Dincler S, Buchmann P (2008) A multivariate analysis of potential risk factors for intra- and postoperative complications in 1316 elective laparoscopic colorectal procedures. Ann Surg 248:259-265

14. Dindo D, Demartines N, Clavien PA (2004) Classification of surgical complications: a new proposal with evaluation in a cohort of 6336 patients and results of a survey. Ann Surg 240(2):205-213

15. Vonlanthen R, Slankamenac K, Breitenstein S, Puhan MA, Muller MK, Hahnloser D, Hauri D, Graf R, Clavien PA (2011) The impact of complications on costs of major surgical procedures: a cost analysis of 1200 patients. Ann Surg 254:907-913

16. Development Core Team (2011) R: a language and environment for statistical computing. R Foundation for Statistical Computing, Vienna, ISBN 3-90005 1-07-0, http://www.R-project.org 
17. Wood SN (2011) Fast stable restricted maximum likelihood and marginal likelihood estimation of semiparametric generalized linear models. J R Stat Soc (B) 73(1):3-36

18. Yang C, Wexner SD, Safar B, Jobanputra S, Jin H, Li VK, Nogueras JJ, Weiss EG, Sands DR (2009) Conversion in laparoscopic surgery: does intraoperative complication influence outcome? Surg Endosc 23:2454-2458

19. Rose J, Schneider C, Yildirim C, Geers P, Scheidbach H, Köckerling F (2004) Complications in laparoscopic colorectal surgery: results of a multicentre trial. Tech Coloproctol 8(Suppl 1):25-28

20. Hubner M, Hahnloser D, Hetzer F, Müller MK, Clavien PA, Demartines N (2007) A prospective randomized comparison of two instruments for dissection and vessel sealing in laparoscopic colorectal surgery. Surg Endosc 21:592-594

21. Heili M, Flowers S, Fowler D (1999) Laparoscopic-assisted colectomy: a comparison of dissection techniques. JSLS 3(1):27-31

22. Morino M, Rimonda R, Allaix ME, Giraudo G, Garrone C (2005) Ultrasonic versus standard electric dissection in laparoscopic colorectal surgery: a prospective randomized clinical trial. Ann Surg 242(6):897-901

23. Neudecker J, Klein F, Bittner R, Carus T, Stroux A, Schwenk W, LAPKON II Trialists (2009) Short- term outcomes from a prospective randomized trial comparing laparoscopic and open surgery for colorectal cancer. Br J Surg 9(12):1458-1467

24. Pikarsky AJ, Saida Y, Yamaguchi, Martinez S, Chen W, Weiss EG, Nogueras JJ, Wexner SD (2002) Is obesity a high-risk factor for laparoscopic colorectal surgery? Surg Endosc 16:855-858
25. Kirchhoff P, Matz D, Dincler S, Buchmann P (2011) Predictive risk factors for intra- and postoperative complications in 526 laparoscopic sigmoid resections due to recurrent diverticulitis: a multivariate analysis. World J Surg 35:677-683

26. Dincler S, Bachmann LM, Buchmann P, Steurer J (2006) Predictors of intra- and postoperative complications in laparoscopic colorectal surgery: results of an expert survey. Dig Surg 23(1-2):110-114

27. Campos FG (2003) Complications and conversions in laparoscopic colorectal surgery: results of a multicenter Brazilian trial. Surg Laparosc Endosc Percutan Tech 13:173-179

28. Bouchard A, Martel G, Sabri E, Schlachta CM, Poulin EC, Mamazza J, Boushey RP (2009) Does experience with laparoscopic colorectal surgery influence intraoperative outcomes? Surg Endosc 23:862-868

29. Langhoff PK, Schultz M, Harvald T, Rosenberg J (2013) Safe laparoscopic colorectal surgery performed by trainees. J Surg Educ 70(1): 144-148

30. Arezzo A, Passera R, Scozzari G, Verra M, Morino M, Langhoff PK, Schultz M, Harvald T, Rosenberg J (2013) Laparoscopy for rectal cancer reduces short-term mortality and morbidity: results of a systematic review and metaanalysis. Surg Endosc 27(5):1485-1502

31. Chapman AE, Levitt MD, Hewett P, Woods R, Sheiner H, Maddern GJ (2001) Laparoscopic-assisted resection of colorectal malignancies: a systematic review. Ann Surg 234:590-606

32. Mroczkowski P, Hac S, Smith B, Schmidt U, Lippert H, Kube R (2012) Laparoscopy in the surgical treatment of rectal cancer in Germany 2000-2009. Colorectal Dis 14(12):1473-1478 